\title{
Nova espécie de Paradaemonia Bouvier (Lepidoptera, Saturniidae, Arsenurinae) do Centro-Sul do Brasil ${ }^{1}$
}

\author{
Amabílio J. A. de Camargo ${ }^{2}$; Olaf H. H. Mielke ${ }^{3} \&$ Mirna M. Casagrande ${ }^{3}$ \\ 1 Contribuição número 1679 do Departamento de Zoologia, Universidade Federal do Paraná. \\ ${ }^{2}$ Embrapa Cerrados. Rodovia BR 020, km 18, Caixa Postal 08223, 73310-970 Planaltina, Distrito Federal, Brasil. \\ E-mail: amabilio@cpac.embrapa.br \\ ${ }^{3}$ Departamento de Zoologia, Universidade Federal do Paraná. Caixa Postal 19020, 81531-980 Curitiba, Paraná, Brasil. \\ Pesquisadordo CNPq. E-mail: omhesp@ufpr.br, mibras@ufpr.br
}

\begin{abstract}
A new species of Paradaemonia Bouvier (Lepidoptera, Saturniidae, Arsenurinae) from the Central Southern Brazil. Paradaemonia Bouvier, 1925 has 14 exclusively neotropical species, widely distributed from Mexico to Argentina. Here a new species, Paradaemonia meridionalis sp. nov., from Central Southern Brazil is described. The species described below is closely related to Paradaemonia castanea (Rothschild, 1907) and P. platydesmia (Rothschild, 1907) group.

KEY WORDS. Neotropical; new moth; taxonomy.
\end{abstract}

RESUMO. Paradaemonia Bouvier, 1925 possui atualmente 14 espécies exclusivamente neotropicais, com distribuição do México até a Argentina. Neste estudo, uma nova espécie, Paradaemonia meridionalis sp. nov., do Centro-Sul do Brasil é descrita. A espécie aqui descrita pertence ao grupo de Paradaemonia castanea (Rothschild, 1907) e $P$. platydesmia (Rothschild, 1907).

PALAVRAS-CHAVE. Neotropical; nova mariposa; taxonomia.

Com a recente descrição de Paradaemonia balsasensis C. Mielke \& Furtado, 2005 e o status revisado de $P$. castanea por RACHeLI (2006), o gênero compreende atualmente 14 espécies. É exclusivamente neotropical, ocorrendo suas espécies do México à Bolívia, Paraguai, norte da Argentina e Brasil (Lemaire 1980, 1996, MielKe \& Furtado 2005).

O objetivo deste trabalho é descrever uma nova espécie do Centro-Sul do Brasil, a qual havia sido equivocadamente considerada por Lemaire (1980) como subespécie de Paradaemonia platydesmia (Rothschild, 1907). A subespécie em questão, Paradaemonia platydesmia castanea (Rothschild, 1907), foi descrita com base em duas fêmeas de Tuís, Costa Rica. A população que ocorre no Centro-Sul do Brasil, apesar de ser um grupo homogêneo, com morfologia externa constante e de apresentar caracteres distintos, foi tradicionalmente considerada como pertencente a esta subespécie (Lemaire 1980: 124).

Lemaire \& Venedictoff (1989) e Lemaire (1996) sinonimizaram $P$. castanea com $P$. platydesmia.

D'Abrera (1995) foi o primeiro autor a mencionar a possibilidade de existirem três espécies envolvidas, isto é, $P$. castanea da Costa Rica, P. platydesmia da Colômbia, Guianas, Bolívia e Brasil (referindo-se provavelmente à parte norte do Brasil), e outra espécie não descrita do sudeste do Brasil, recomendando a sua descrição.
Mais recentemente RACHeLI (2006) removeu P. castanea da sinonímia de $P$. platydesmia elevando-a novamente ao status de espécie como havia sido proposto originalmente por RothSCHILD (1907) e ampliando a distribuição geográfica para o Peru, de onde menciona dois exemplares (machos) do departamento de Junin (Chanchamayo, Puente S. Felix).

A confusão gerada deve-se ao conhecimento de somente dois exemplares fêmeas, mencionados na descrição original de P. castanea, ambos provenientes de Tuís, Costa Rica, país considerado como bem inventariado do ponto de vista lepidopterológico, no entanto, no momento são conhecidos mais 12 exemplares machos da Costa Rica (coleção INBio) e que coincidem com os caracteres dos exemplares femininos coletados em Tuís. Logo a eventual suspeita de erro de etiquetagem não procede.

Os exemplares utilizados neste artigo são os mencionados na série tipo de Paradaemonia meridionalis sp. nov. e das espécies utilizadas nas comparações como segue: Paradaemonia platydesmia: 1 macho 18-I-1979, BrasiL, Pará: Santa Isabel, DZ 8.914; 1 fêmea, 21-IX-1963, Brasil, Amapá: Serra do Navio, ICOMI, D'Almeida leg., DZ 8.922; 1 macho, III-2001, GuiAnA Francesa: Kaw, OM 67.460. Paradaemonia castanea: 1 macho, IV-1990, Costa Rica, Heredia: N.P. 400-600 m, Braulio Carillo, Est. El Ceibo, C. Chaves leg., 527700-256500, INBio 210315, 
doado à coleção Pe Jesus Santiago Moure, Setor de Ciências Biológicas, Universidade Federal do Paraná, Departamento de Zoologia, DZ 8.915.

Os acrônimos das coleções são os seguintes: (CPAC) Coleção EMBRAPA Cerrados, Planaltina, Distrito Federal, Brasil; (DZUP) Coleção Pe Jesus Santiago Moure, Setor de Ciências Biológicas, Universidade Federal do Paraná, Curitiba, Paraná, Brasil; (INBio) Instituto Nacional de Biodiversidad, Santo Domingo, Heredia, Costa Rica; (INPA) Instituto Nacional de Pesquisas da Amazônia, Manaus, Amazonas, Brasil; (OM) Coleção Olaf H.H. Mielke, Curitiba, Paraná, Brasil.

\section{Paradaemonia meridionalis sp. nov.}

\section{Figs 1-4, 11-15}

Diagnose. Esta nova espécie, juntamente com P. platydesmia e $P$. castanea constituem um grupo de espécies que se caracteriza pela envergadura das asas e pela faixa sub-basal das asas anteriores estreita. Paradaemonia meridionalis possui a face dorsal das asas anteriores castanho avermelhado e ausência de escamas brancas no vértice, entre e atrás das antenas em ambos os sexos. Genitália masculina com harpe sobrepondo a ampola e expansão dorsal do unco menor que seu comprimento.

Descrição. Macho (Figs 1, 2): Holótipo com asa anterior de 7,3 cm de comprimento e envergadura de $13,5 \mathrm{~cm}$. Parátipos. Asa anterior de 7,0 a 7,5 cm de comprimento e envergadura de 12,6 a $14,5 \mathrm{~cm}$.

Coloração geral do corpo e face dorsal de ambas as asas castanho-avermelhado. Vértice, entre e atrás das antenas, sem faixa de escamas brancas. Asa anterior com faixa sub-basal mais estreita quando comparada à de $P$. platydesmia e $P$. castanea e margem externa logo abaixo da expansão apical, com ondulações discretas. Face ventral de ambas as asas castanho avermelhado menos intenso que aquele da face dorsal e com os dois terços basais mais claros que a porção marginal. Nas asas posteriores veias $\mathrm{M}_{3}-\mathrm{CuA}$ partem separadamente da célula discal.

Genitália. Bifurcação do unco em ângulo fechado, tegumento esclerotinizado ligando a juxta com a transtila, gnato robusto e fortemente esclerotinizado, vesica falciforme e longa, ampola de aspecto arredondado, harpe parcialmente sobrepondo a ampola e elevação dorsal do unco menor que o comprimento (Figs 13-15).

Fêmea (Figs 3-4): Asa anterior entre 7,4 $\mathrm{cm}$ e 7,9 $\mathrm{cm}$ de comprimento e envergadura entre $13,0 \mathrm{~cm}$ a $14,9 \mathrm{~cm}$. Coloração semelhante ao macho, com asas mais arredondadas e maior envergadura. Face dorsal da asa posterior com a metade basal clara pela ausência de escamas escuras na área anal, presentes nas outras duas espécies do grupo.

Holótipo macho com as seguintes etiquetas: /Holotypus/ II-2005, Rio Natal, São Bento do Sul, Santa Catarina, Brasil, 500 m, I. Rank leg./ Holotypus Paradaemonia meridionalis Camargo, Mielke \& Casagrande/ DZ 9.063/. Depositado na coleção Pe. Jesus Santiago Moure, Departamento e Zoologia, Setor de Ciências Biológicas, Universidade Federal do Paraná.
Alótipo fêmea com as seguintes etiquetas: /Allotypus/ 5XI-1973, São Bento do Sul, Santa Catarina, Brasil, J. Weiss leg./ Ex Col. Gagarin/ Allotypus Paradaemonia meridionalis Camargo, Mielke \& Casagrande/ DZ 9.071/. Depositado na coleção Pe. Jesus Santiago Moure, Departamento e Zoologia, Setor de Ciências Biológicas, Universidade Federal do Paraná.

Parátipos. Brasil, Distrito Federal: Planaltina, 1 macho 19X-1976, V.O. Becker leg., CPAC 1679; Espírito Santo: Cachoeira de Santa Teresa, 1 macho 8-X-1961, Aristides Gentili leg., ex coleção Gagarin, DZ 10.100. Rio de Janeiro: Petrópolis, 1 macho, 15-X-1943, Gagarin leg., ex coleção Gagarin, DZ 10.344, 1 macho, 16-X-1955, D'Almeida leg., ex coleção D'Almeida 14.271, DZ 10.051, 1 macho, 21-II-1960, Gagarin leg., ex coleção Gagarin, DZ 10.432, 1 macho, 19-X-1960, Gagarin leg., ex coleção Gagarin, DZ 9.001, 1 macho, 21-X-1960, Gagarin leg., ex coleção Gagarin, DZ 10.108, 1 macho, 2-XI-1961, Gagarin leg., ex coleção Gagarin, DZ 9.009, Petrópolis (Alto da Serra), 1 macho, 2-XI-1956, Arlindo leg., ex coleção Richard Frey, DZ 10.147, Petrópolis (Independência, 900 m), 1 macho, 17-IX-1939, Gagarin leg., ex coleção Gagarin, DZ 10.364, 1 fêmea, 23-IX-1939, Gagarin leg., ex coleção Gagarin, DZ 8.929, 1 macho, 20-X-1939, Gagarin leg., ex coleção Gagarin, DZ 10.194, Petrópolis (Parque São Vicente, 900 m), 1 fêmea, 29-IX-1959, Gagarin leg., ex coleção Gagarin, DZ 8.945, 1 macho, 3-IX-1962, Gagarin leg., ex coleção Gagarin, DZ 10.306, Petrópolis (Parque São Vicente, 920 m), 1 macho, 29-I1963, Gagarin leg., ex coleção Gagarin, DZ 10.245; São Paulo: Salesópolis (Reserva Boracéia, 950 m), 1 macho, 1 fêmea, 13, 14XII-1941, D'Almeida leg., ex coleção D'Almeida 14.272 e 14.273, DZ 10.121, DZ 9.079, Campos do Jordão (Umuarama, 1800 m), 1 macho, 3-15-II-1937, Gagarin leg., ex coleção Gagarin, DZ 10.309, 1 fêmea, 8-15-IV-1937, Gagarin leg., ex coleção Gagarin, DZ 9.087; Paraná: Piraquara (Banhado, 850 m), 1 macho, 21-XI1971, Leonart leg., DZ 8.985, Tijucas do Sul (Voçoroca, 850 m), 1 macho, 19-I-1972, Mielke leg., INBio, 1 fêmea, 17-II-1977, Mielke leg., DZ 8.937, 1 fêmea, 28-II-1979, Mielke leg., INBio, Piraquara (850 m), 2 machos, 23-II-2003, Camargo leg., CPAC 20485, CPAC 20486, Piraquara (Manaciais da Serra, 850 m), 1 macho, 16-XII2006, Rafael \& Melo leg., INPA, Curitiba (900 m), 1 macho, XI1944, H. Riesenberg leg., ex coleção F. Justus Jr, DZ 10.407, Campo Largo (900 m), 1 macho, 7-X-2003, Novak leg., DZ 10.150, Ponta Grossa (900 m), 1 macho, XI-1957, Justus leg., ex coleção F. Justus Jr, DZ 10.343, Guarapuava (1000 m), 1 macho, 1-II1971, Laroca \& Jansen leg., DZ 8.993, Foz do Iguaçu (Parque Nacional do Iguaçu), 2 fêmeas, 7-XII-1966, 11-XII-1966, D. Zoo. U.F.P. leg., DZ 8.913, DZ 9.025; Santa Catarina: São Bento do Sul (850 m), 1 macho, 20-II-1970, J. Weiss leg., ex coleção Gagarin, DZ 10.119, 1 fêmea, 27-X-1972, J. Weiss leg., ex coleção Gagarin, DZ 8.953, São Bento do Sul (700 m), 1 fêmea, 21-I-1974, J. Weiss leg., ex coleção Gagarin, DZ 9.095, 1 macho, 17-IX-1974, J. Weiss leg., ex coleção Gagarin, DZ 9.111, São Bento do Sul, ( 700 m), 1 macho, 13-X-1974, ex coleção Gagarin, J. Weiss leg., DZ 10.404, 1 macho, 23-XII-1974, ex coleção Gagarin, J. Weiss leg., DZ 10.467, São Bento do Sul, 1 macho, 28-III-1975, ex coleção 


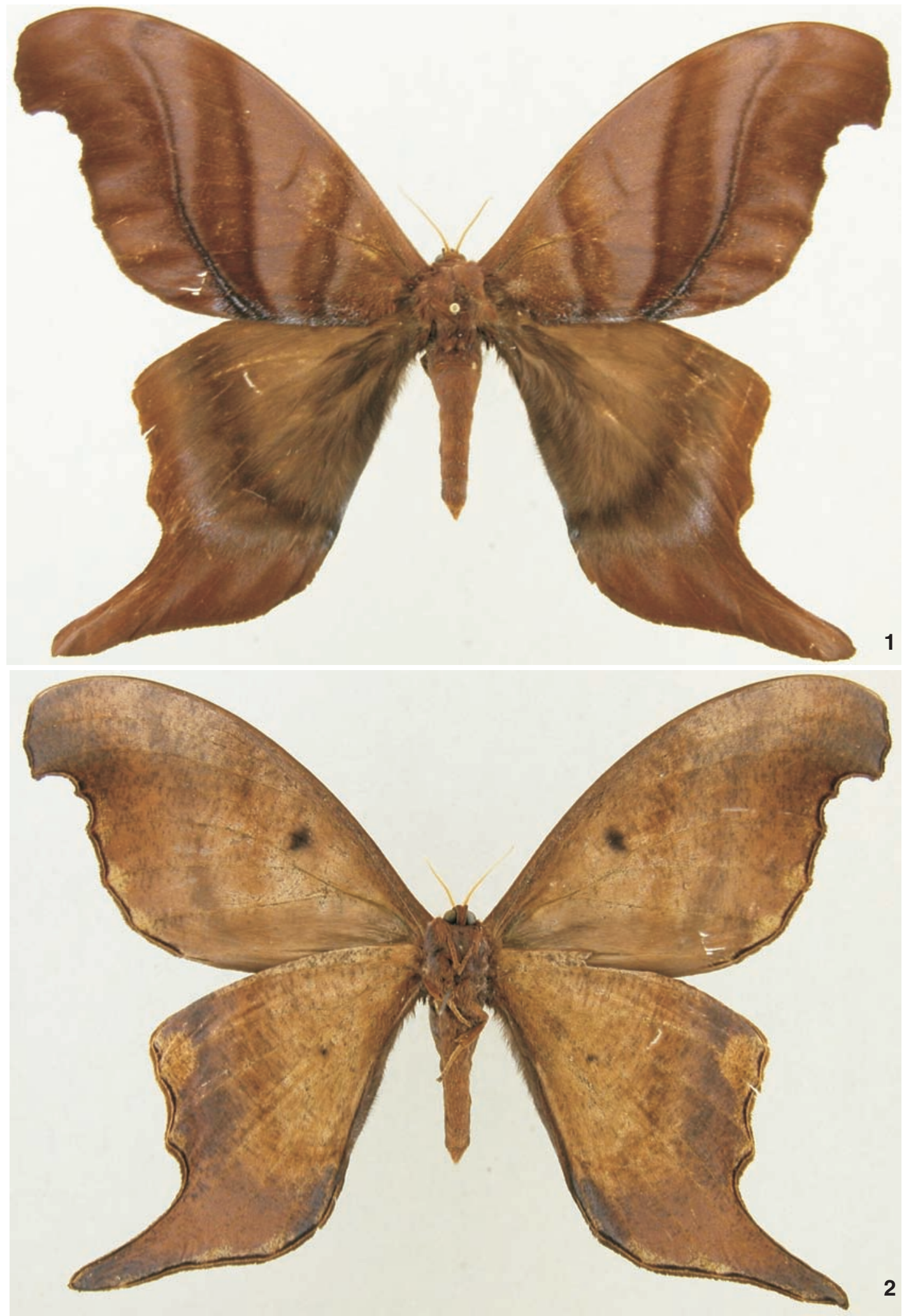

Figuras 1-2. Paradaemonia meridionalis sp. nov., holótipo macho: (1) dorsal; (2) ventral. 

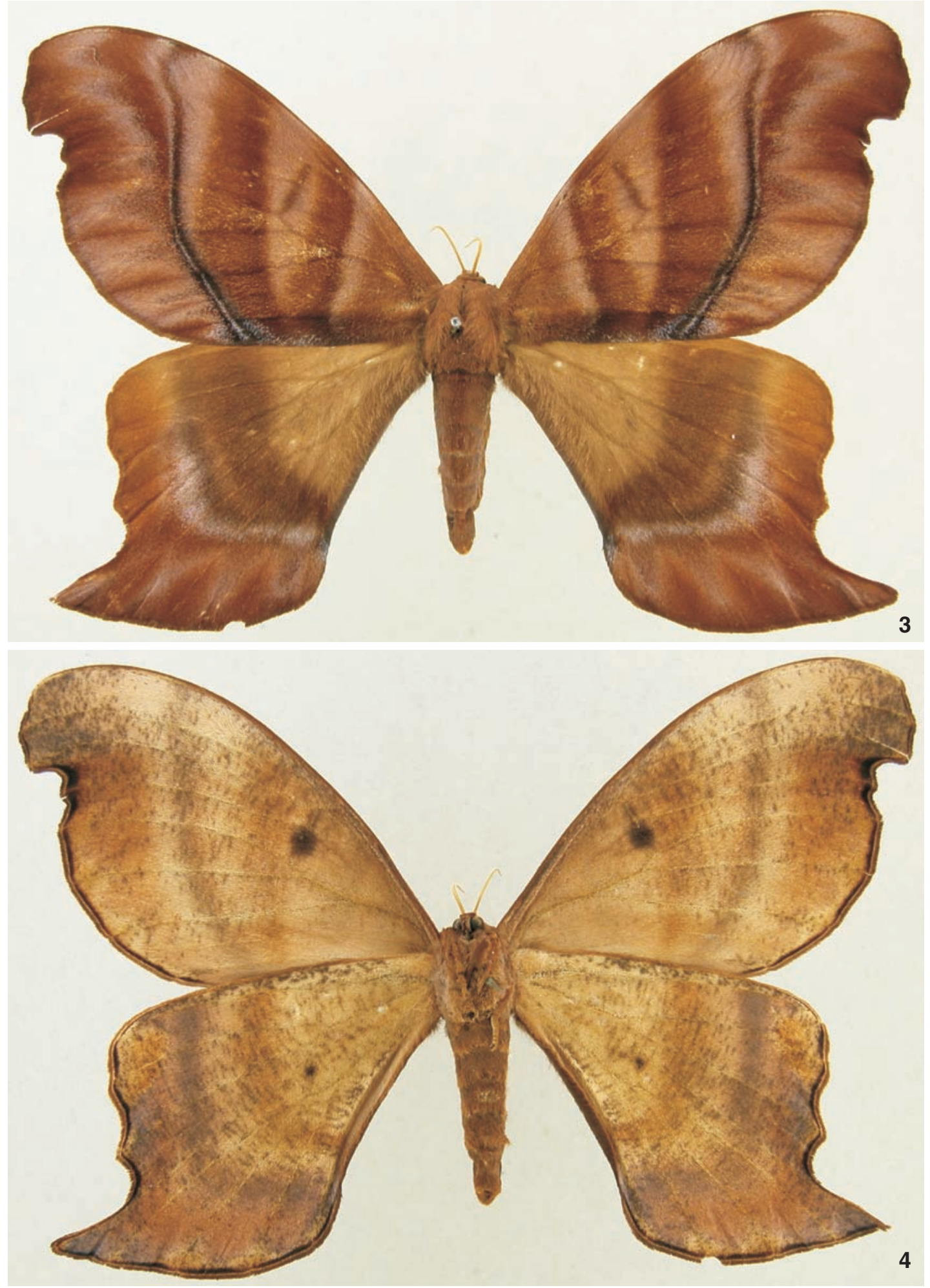

Figuras 3-4. Paradaemonia meridionalis sp. nov., alótipo fêmea: (3) dorsal, (4) ventral.

Revista Brasileira de Zoologia 24 (4): 1131-1138, dezembro 2007 

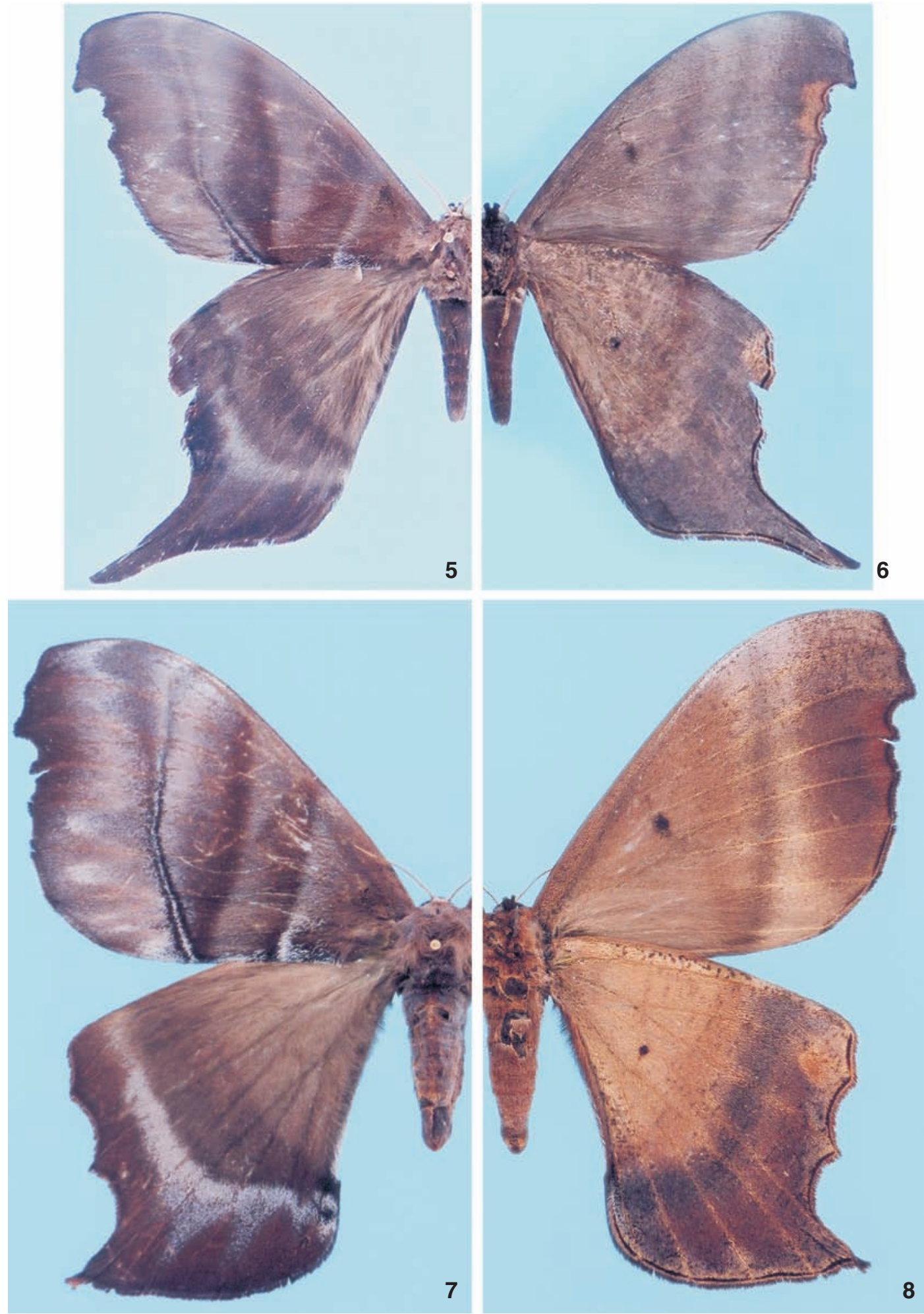

Figuras 5-8. Paradaemonia platydesmia: (5-6) macho, 18-I-1979, Santa Isabel, Pará, Brasil: (5) dorsal; (6) ventral; (7-8) fêmea, 3-VII1999, Piste de Kaw, PK 36, Guiana Francesa: (7) dorsal; (8) ventral. 

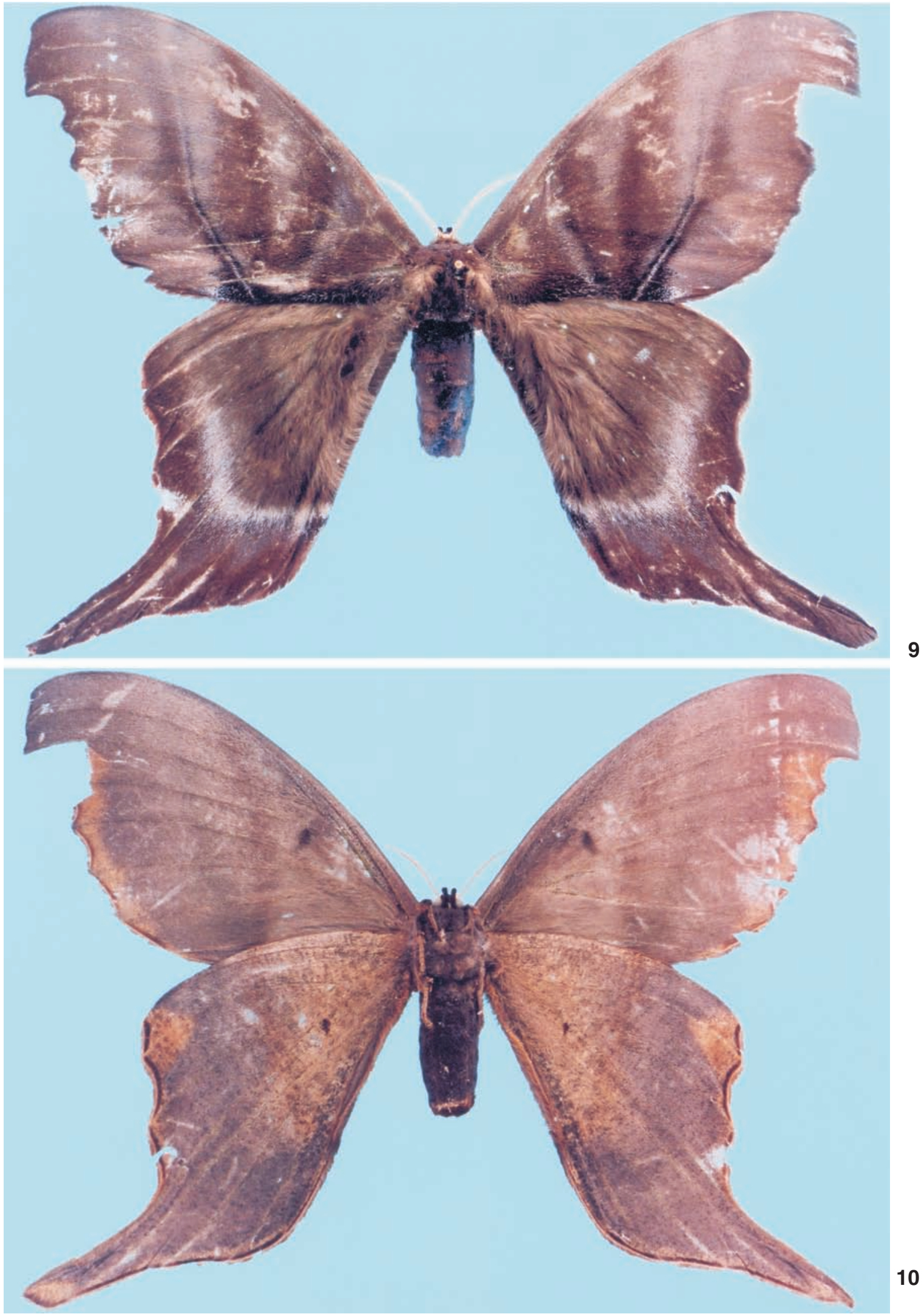

Revista Brasileira de Zoologia 24 (4): 1131-1138, dezembro 2007 

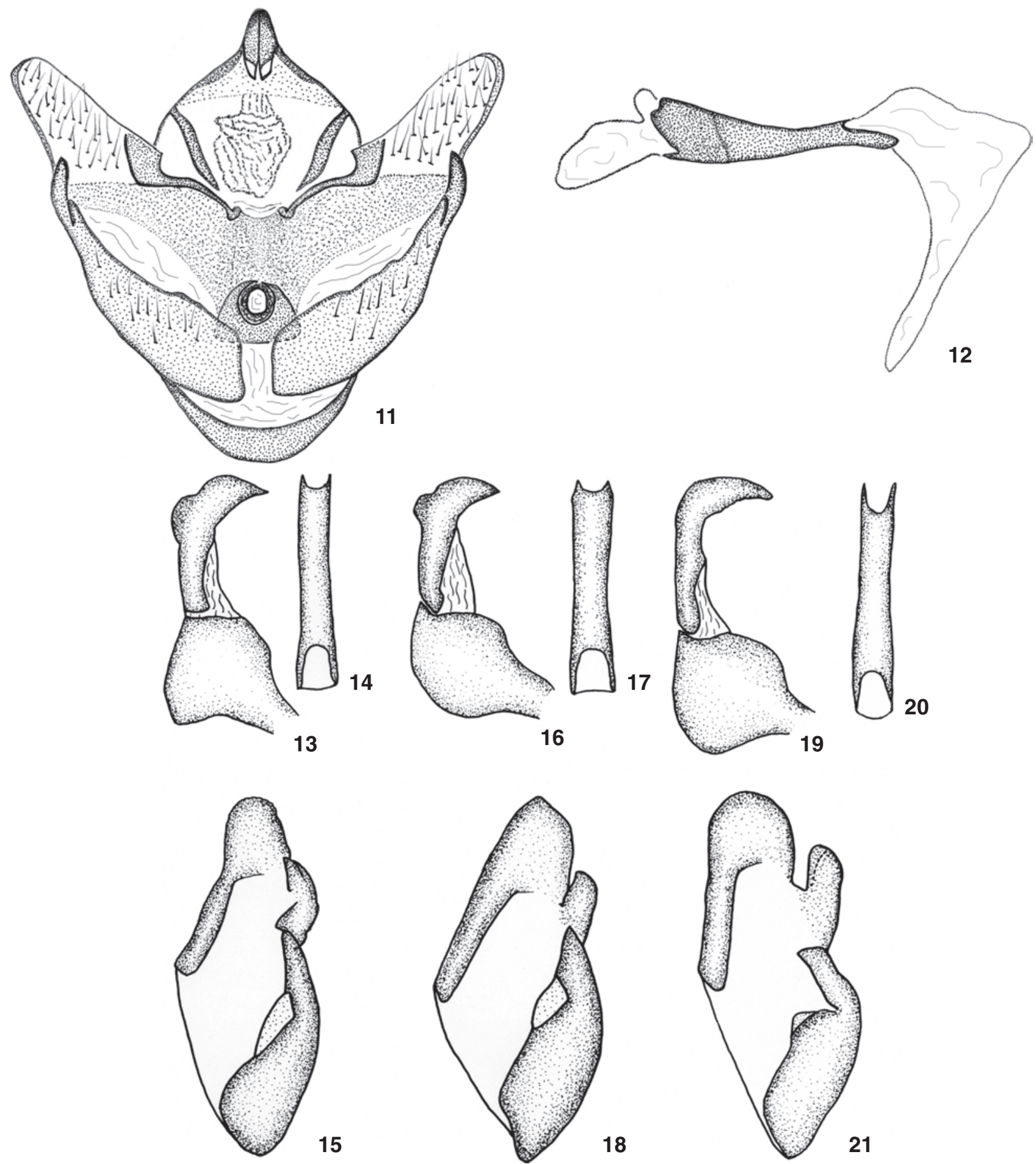

Figuras 9-21. (9-10) Fotografias na página à esquerda: Paradaemonia castanea, macho, IV-1990, Est. El Ceibo, Braulio Carillo, N.P. 400600 m, Heredia, Costa Rica, C. Chaves leg.: (9) dorsal; (10) ventral; (11-21) Genitálias masculinas. (11-15) Paradaemonia meridionalis sp. nov.: (11) vista ventral; (12) vista lateral do edeago com vesica evertida; (13) vista lateral do tegumen e unco; (14) vista dorsal do edeago; (15) face interna da valva direita; (16-18) Paradaemonia platydesmia: (16) vista lateral do tegumen e unco; (17) vista dorsal do edeago; (18) face interna da valva direita; (19-21) Paradaemonia castanea: (19) vista lateral do tegumen e unco; (20) vista dorsal do edeago; (21) face interna da valva direita. 
Gagarin, DZ 9.055, São Bento do Sul (Rio Natal, 500 m), 1 fêmea, 10-X-1981, Rank leg., DZ 9.103, São Bento do Sul (Rio Natal, 550 m), 1 fêmea, 21-XI-1987, C. Mielke leg., CPAC 20487, 2 machos, I-2005, Rank leg., OM 67.308, OM 67.567, São Bento do Sul (Rio Vermelho, 850 m), 1 fêmea, 20-X-1979, Rank leg., DZ 8.961, São Bento do Sul (Rio Vermelho, 850 m), 1 fêmea, 18XII-1979, Rank leg., DZ 8.921, 1 fêmea, 12-XII-1980, Rank leg., DZ 8.977; Rio Grande do Sul: Cambará do Sul (Estância Cambará, 1040 m), 1 macho 1-4-I-2006, C. Mielke leg., DZ 9.035.

Discussão. O status de espécie é plenamente justificado, visto que $P$. meridionalis do centro-sul do Brasil contrasta com $P$. platydesmia da região amazônica e com $P$. castanea, da Costa Rica e Peru, pela coloração castanho-avermelhada uniforme e constante em ambos os sexos, maior envergadura e ausência da faixa clara entre e atrás das antenas.

Em P. platydesmia (Figs 5-8) a asa anterior do macho mede $6,0 \mathrm{~cm}$, a coloração alar é castanho-acinzentada, faixa sub-basal da asa anterior larga, margem externa da asa anterior com ondulações mais evidentes; vértice com faixa transversal branca nítida no macho, às vezes menos destacada na fêmea. Paradaemonia castanea (Figs 9 e 10) assemelha-se a P. platydesmia na coloração da asa que também é castanho-acinzentada, pelas ondulações na margem externa da asa anterior e pelo vértice com faixa branca, e assemelha-se à $P$. meridionalis pela faixa sub-basal estreita e pelo comprimento da asa anterior.

Ventralmente $P$. meridionalis difere de ambas pela coloração castanho-avermelhada menos intensa que na face dorsal e com os dois terços basais mais claros que a porção marginal. Em ambas, $P$. platydesmia e $P$. castanea, a face ventral apresenta coloração castanho-acinzentada com tonalidades menos intensas do que na face dorsal e com a porção basal mais clara.

Quanto à venação, nas asas posteriores do macho, as veias $\mathrm{M}_{3}$ e CuA 1 partem separadamente da célula discal, enquanto que em $P$. platydesmia e $P$. castanea estas veias tem origem comum.

Em fêmeas de $P$. meridionalis, nas asas posteriores a metade basal é clara e em $P$. castanea (ver figura em D'Abrera 1995) esta área apresenta-se pouco diferenciada. As fêmeas de $P$. platydesmia (Figs 7 e 8) apresentam coloração dorsal semelhante aos machos, porém, com a metade basal da asa posterior mais clara e com a faixa branca no vértice menos nítida.

A genitália de $P$. meridionalis (Figs 11-15) difere da de $P$. platydesmia e de P. castanea, ver Lemaire (1980) e Mielke \& FurtaDo (2005). Em P. platydesmia a ampola é mais afilada, a harpe encobre a ampola apenas na sua base e a expansão dorsal do unco igual ao seu comprimento (Figs 16-18). Difere de P. castanea onde a ampola é mais larga, harpe distanciada da ampola, sem expansão dorsal no unco e a terminação do edeago, em vista dorsal, fortemente côncava (Figs 19-21).

Distribuição geográfica. Abrange a área compreendida pelo Distrito Federal e estados de Goiás (Ponte Funda, Vianópolis - Lemaire 1980), Espírito Santo ao Rio Grande do Sul em altitudes que variam desde o nível do mar até $1800 \mathrm{~m}$.

Planta hospedeira: A espécie utiliza em São Bento do Sul, Santa Catarina, Symplocos tenuifolia Brand (Symplocaceae) como planta hospedeira.

Etimologia: O nome meridionalis se refere à sua distribuição meridional, em relação às espécies próximas: $P$. platydesmia e $P$. castanea.

\section{AGRADECIMENTOS}

A Isidro Chacon, INBio, Costa Rica, pelas informações sobre o número de exemplares de Paradaemonia castanea na coleção do INBio e pela doação de um exemplar macho à Coleção Pe Jesus Santiago Moure; a Gert G. Hatschbach, Museu Botânico Municipal de Curitiba, pela identificação da planta hospedeira; e a Ivo Rank pelas informações sobre a planta hospedeira.

\section{REFERÊNCIAS BIBLIOGRÁFICAS}

D'Abrera, B. 1995. Saturniidae Mundi. Saturniid Moths of the World. Part 1, based on the Collections of the Natural History Museum in London, (British Museum (Natural History)). Keltern, Germany, Automeris Press, 177p.

Lemaire, C. 1980. Les Attacidae Américains (= Saturniidae). Arsenurinae. Neuilly-sur-Seine, Édition C. Lemaire, 199p.

Lemaire, C. 1996. Saturniidae, p. 28-49. In: J.B. Heppner (Ed.). Atlas of Tropical Lepidoptera. Part 4B. Gainesville, Scientific Publishers, XLIX+87p.

Lemaire, C. \& N. Venedictoff, 1989. Catalogue and biogeography of the Lepidoptera of Ecuador. I - Saturniidae, with a description of a new species of Meroleuca Packard. Bulletin of the Allyn Museum 129: 1-60.

Mielke, C.G.C. \& E. Furtado. 2005. Paradaemonia balsasensis sp. nov. da Serra do Penitente, Balsas, Maranhão, Brasil (Lepidoptera, Saturniidae, Arsenurinae). Revista Brasileira de Zoologia 22 (3): 653-655.

Racheli, L. 2006. List of the Arsenurinae of Peru with taxonomic notes on Titaea raveni (Johnson \& Michener, 1948) stat. rev. and Parademonia castanea (Rothschild, 1907) stat. rev. (Lepidoptera, Saturniidae). Galathea 22 (1): 41-47.

RotнsсhiLD, W. 1907. New American Saturniidae and Ceratocampidae. Novitates Zoologicae 14: 413-432.

Recebido em 30.III.2007; aceito em 27.XI.2007. 